\title{
Biomarkers Related to Endothelial Dysfunction and Vascular Cognitive Impairment: A Systematic Review
}

\author{
Rui Kleber Martins-Filho ${ }^{\mathrm{a}}$ Maria Clara Zotin ${ }^{\mathrm{b}} \quad$ Guilherme Rodrigues $^{\mathrm{a}}$ \\ Octavio Pontes-Neto ${ }^{a}$ \\ aDepartment of Neurosciences and Behavioural Sciences, Hospital das Clínicas - Ribeirão Preto Medical School, \\ University of São Paulo, Ribeirão Preto, Brazil; 'b Department of Internal Medicine, Radiology Division, Hospital das \\ Clínicas - Ribeirão Preto Medical School, University of São Paulo, Ribeirão Preto, Brazil
}

\section{Keywords}

Endothelial dysfunction - Vascular cognitive impairment .

Vascular dementia. Cerebral small vessel disease

\begin{abstract}
Introduction: The damage in the endothelium and the neurovascular unit appears to play a key role in the pathogenesis of vascular cognitive impairment (VCl). Although there have been many advances in understanding the physiopathology of this disease, several questions remain unanswered. The association with other degenerative diseases and the heterogeneity of its clinical spectrum establish a diagnostic problem, compromising a better comprehension of the pathology and halting the development of effective treatments. The investigation of biomarkers is an important movement to the development of novel explicative models and treatment targets involved in VCl. Methods: We searched MEDLINE considering the original research based on VCl biomarkers in the past 20 years, following prespecified selection criteria, data extraction, and qualitative synthesis. Results: We reviewed 42 articles: 16 investigated plasma markers, 17 analyzed neuropathological markers, 4 studied CSF markers, 4 evaluated neuroimaging markers (ultrasound and MRI),
\end{abstract}

and 1 used peripheral Doppler perfusion imaging. Conclusions: The biomarkers in these studies suggest an intrinsic relationship between endothelial dysfunction and $\mathrm{VCl}$. Nonetheless, there is still a need for identification of a distinctive set of markers that can integrate the clinical approach of $\mathrm{VCl}$, improve diagnostic accuracy, and support the discovery of alternative therapies.

(c) 2020 S. Karger AG, Basel

\section{Introduction}

Small vessel disease (SVD) is a clinical-radiological syndrome caused by a disorder in perforating cerebral arterioles, capillaries, and venules, resulting in lesions of cerebral white and deep gray matter [1]. It is responsible for about $20 \%$ of strokes and $45 \%$ cases of dementia in the world $[2,3]$. Endothelial cells appear to have a pivotal role in the pathogenesis of cerebral SVD and vascular dementia $(\mathrm{VaD})$. Endothelial dysfunction (ED) may contribute to SVD pathology through multiple mechanisms, such as blood-brain barrier (BBB) damage, decrease in wholebrain or tissue-resting cerebral blood flow, loss of cerebral vasoreactivity, and increase in intracranial pulsatility [4]. karger@karger.com

(C) 2020 S. Karger AG, Basel

www.karger.com/dem

Karger ${ }^{\prime}=$
Rui Kleber Martins-Filho

Department of Neurosciences and Behavioural Sciences, Hospital das Clínicas Ribeirão Preto Medical School, University of São Paulo

Bernardino de Campos 1000, Centro, Ribeirão Preto 14015-130 (Brazil) ruikleber@usp.br 
Fig. 1. Flow of information through the different phases of this systematic review. VCI, vascular cognitive impairment.

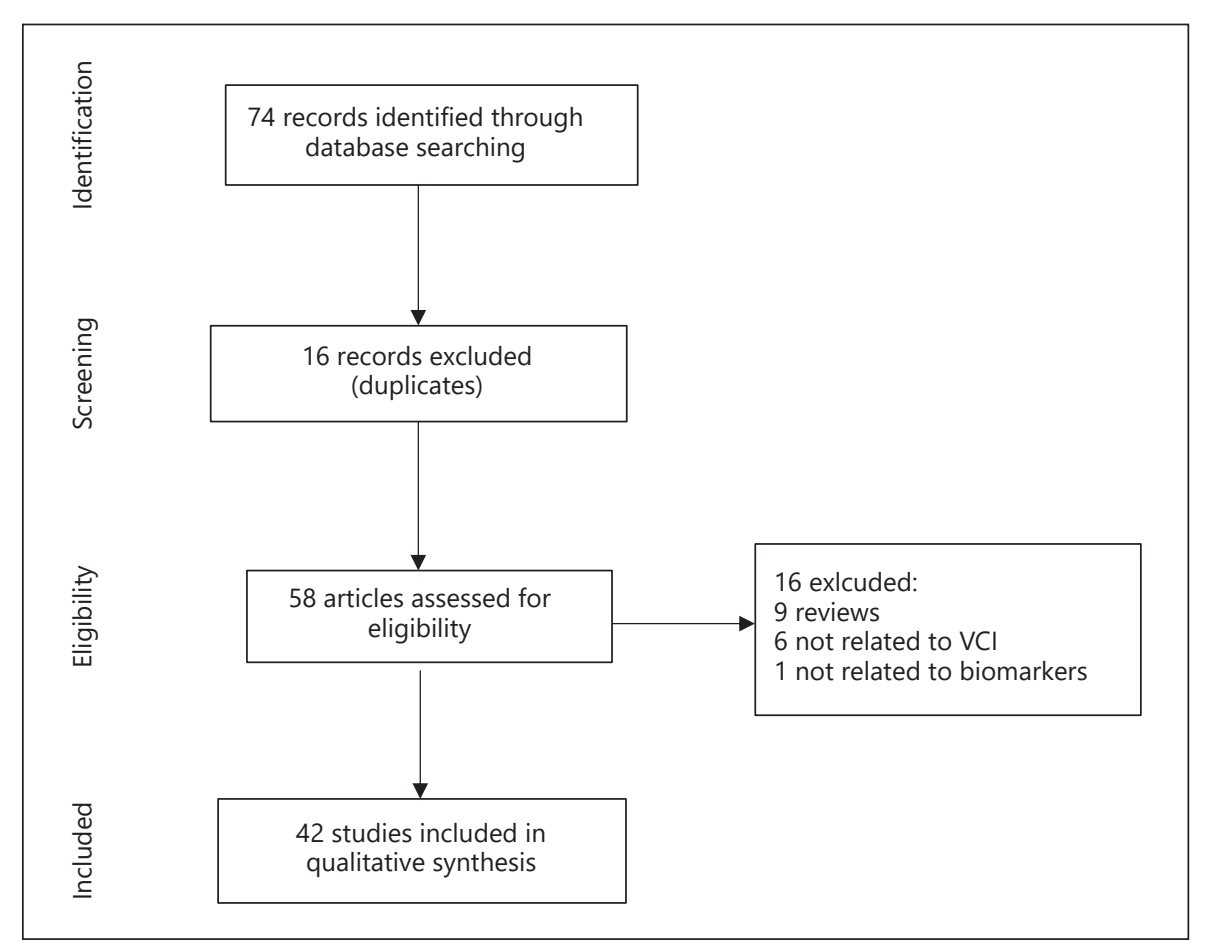

Moreover, ED may represent a potential link between cerebral SVD and Alzheimer's pathology. For example, the leakage of fibrinogen through vessel walls contributes to amyloid-beta $(\mathrm{A} \beta)$ plaque formation [1] and disrupted transport across the BBB plays a significant role in determining $A \beta$ concentrations in the central nervous system (CNS) $[5,6]$.

Recently, Alzheimer's Disease-Related Dementias Summit set the study of small vessel VCI biomarkers as a research priority [7]. Considering that a thorough review analyzing markers of ED in patients with VCI is lacking, we carried out a systematic review on biomarkers of ED in VCI studies undertaken in the last 20 years.

\section{Methods}

Search Strategy, Study Selection, and Data Acquisition

We searched MEDLINE for studies investigating ED and VCI with the following free text and Medical Subject Headings (MeSH): "endothelium" or "endothelial" and "cognitive impairment" or "dementia." We limited the search to articles written in English, with human adults as subjects and published in the last 20 years (search period between January 1, 1999, and December 31, 2019).

According to the Preferred Reporting Items for Systematic Reviews and Meta-Analyses (PRISMA) statement [8], 2 investigators (R.K.M.F. and G.R.) searched MEDLINE independently and compared their results. Differences in the screening phase were re- solved by consensus. Three investigators (R.K.M.F., M.C.Z., and G.R.) carried out data extraction independently. They stored the variables in a spreadsheet especially developed for this review, tabulating the following data: number of participants, population studied, study design, biomarker of interest, source of biomarker, and main results.

\section{Results}

The combined terms yielded 74 articles: "endothelial" and "vascular cognitive impairment" - 5 articles; "endothelium" and "vascular cognitive impairment" - 3 articles; "endothelial" and "vascular dementia" - 49 articles; and "endothelium" and "vascular dementia" - 17 articles. After excluding studies in duplicates, 58 articles remained. In sequence, R.K.M.F. and G.R. independently reviewed all identified abstracts and excluded articles that met any of the following criteria: (a) articles not related to VCI; (b) no original research (i.e., reviews, editorials, and letters); (c) research not focused on endothelial biomarkers. Again, any discrepancies were resolved by consensus.

From 58 articles initially identified, we excluded 16 papers in this second phase (9 reviews, 6 not related to VCI, and 1 did not study biomarkers) as shown in Figure 1. From the 42 articles included in the qualitative synthesis, 
Table 1. Main biomarkers related to ED in VCI [9, 12-21, 23-30, 35-37, 40, 41, 44, 45, 48, 53-55]

Category

Reference

$B B B$ dysfunction markers

$\uparrow \mathrm{CSF}:$ plasma albumin ratio

Adhesion molecules: $\uparrow$ ICAM-1, VCAM-1, and sICAM-1

[9]

Plasmatic inflammatory markers: $\uparrow$ platelet factor IV, CD40 ligand, homocysteine, interleukin-6, TNF- $\alpha$, and

C-reactive protein

Endothelial function mediators: $\uparrow$ endothelin-1, natriuretic atrial peptide, von Willebrand factor, and angiopoietin-2; $\downarrow$ NO synthase

Endothelial assembly: $\uparrow$ collagen types I and IV, kallikrein 6, occludin, and claudin

$[9,12,13]$

$[13-16]$

[17-21]

$[23-27]$

Perfusional and hemodynamic markers

$\uparrow$ VEGF and TGF- $\beta$

$[9,29]$

No change: VEGF

$[28,30]$

Neoangiogenesis

$[35,36]$

$\downarrow$ MAG:PLP1 ratio (hypoperfusion)

[37]

$\uparrow$ ANGPTL4, ET-1, thrombomodulin, and dihydroxyeicosatrienoic acid

$[40,41,44,55]$

Vasoreactivity markers

$\downarrow$ Cerebral vasoreactivity to $\mathrm{CO}_{2}$

$\downarrow$ Peripheral vasoreactivity

Other markers

$\uparrow \beta$-amyloid 40 (vasoconstrictive effect)

$[54]$

$\downarrow$ CD34 and CD133 (endothelial progenitor cells)

[56]

ED, endothelial dysfunction; VCI, vascular cognitive impairment; BBB, blood-brain barrier; CSF, cerebral spinal fluid; ICAM-1, brain endothelial intercellular adhesion molecule 1; VCAM-1, vascular cell adhesion molecule 1; sICAM-1, plasma level of soluble intercellular adhesion molecule 1; VEGF, vascular endothelial growth factor; TGF- $\beta$, transforming growth factor $\beta$; MAG, myelin-associated glycoprotein; PLP1, proteolipid protein 1; ANGPTL4, angiopoietin-like 4; TNF- $\alpha$, tumor necrosis factor-alpha; NO, nitric oxide; ET-1, endothelin-1; DBF, dermal blood flow; NfL, neurofilament light chain.

16 investigated plasma markers, 17 analyzed neuropathological markers, 4 studied CSF markers, 4 evaluated neuroimaging markers (ultrasound and MRI), and 1 dealt with peripheral Doppler perfusion imaging. The main findings of each study are summarized in online suppl. Table 1; for all online suppl. material, see www.karger. com/doi/10.1159/000510053.

\section{Discussion}

\section{Summary of Evidence}

This systematic review aims to describe biomarkers that target pathways linking ED with cerebral SVD and VCI. We divided such markers into those related to BBB dysfunction, those related to perfusional and hemodynamic changes, and those that assess cerebrovascular and peripheral reactivity. Table 1 summarizes the most important known facts to each category. Some of the main markers are represented in a schematic model illustrated in Figure 2.

\section{Blood-Brain Barrier Dysfunction}

A functional $\mathrm{BBB}$ depends on the adequate interaction between the many components of the neurogliovascular unit to regulate the transit of fluid and nutrients between intravascular and interstitial spaces, maintaining CNS homeostasis [9]. Biochemical markers, found in the CSF and the plasma, are related to BBB dysfunction in patients with VCI.

\section{CSF/Plasma Albumin Ratio}

The ratio between albumin levels in CSF and plasma (Qalb), though not specific, represents a commonly used method to measure the degree of disruption of BBB. Janelidze et al. [9] found increased Qalb in participants with multiple forms of dementia, although only slightly higher values were demonstrated among subjects with 


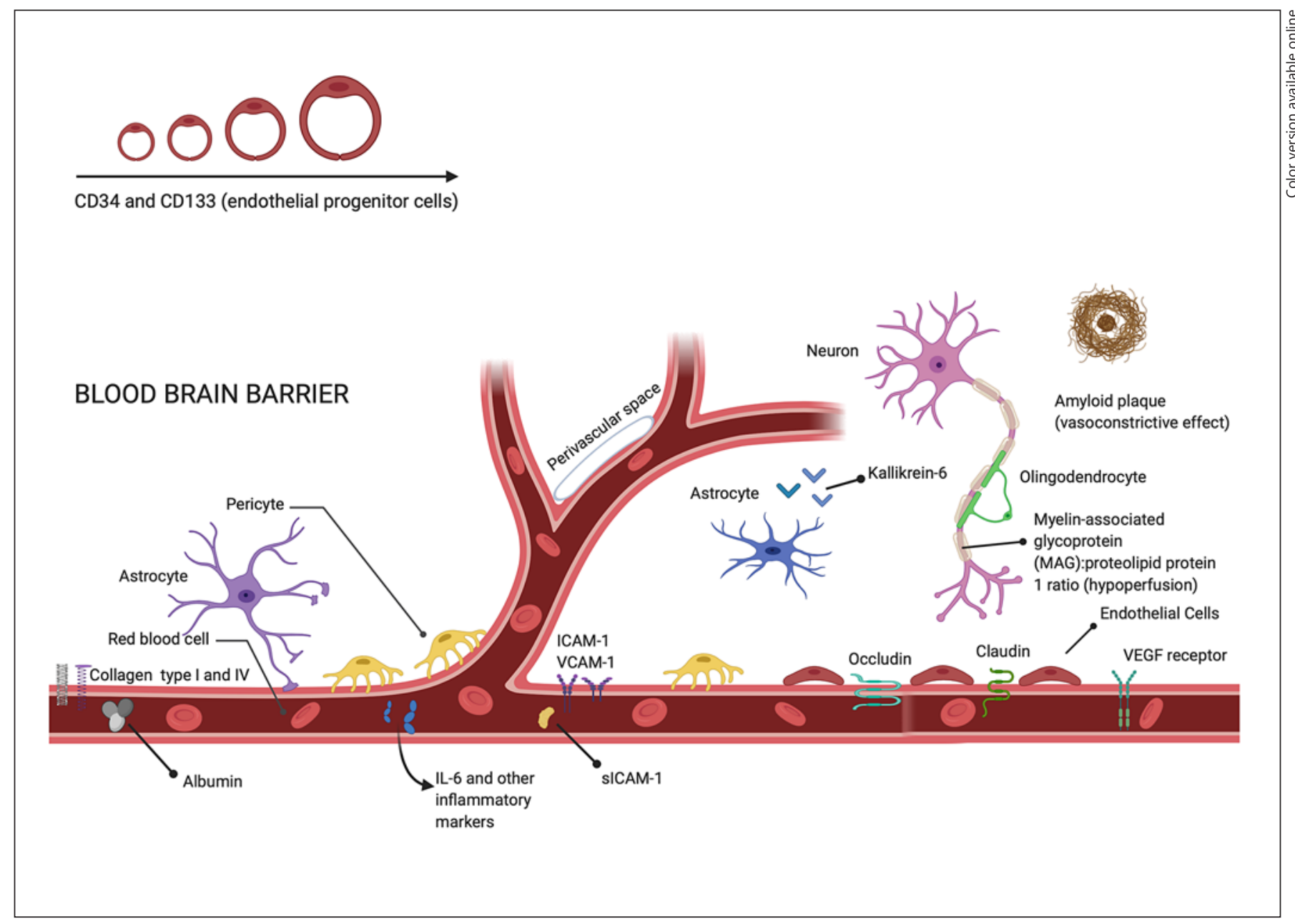

Fig. 2. Schematic model illustrating some of the key markers covered in this review (customized with BioRender). ICAM-1, brain endothelial intercellular adhesion molecule 1; VCAM-1, vascular cell adhesion molecule 1; sICAM-1, plasma level of soluble intercellular adhesion molecule 1; VEGF, vascular endothelial growth factor; MAG, myelin-associated glycoprotein; PLP1, proteolipid protein 1.

$\mathrm{VaD}$. These findings suggest that BBB dysfunction may be a common feature in different dementia types. Interestingly, Qalb was associated with diabetes mellitus (DM) and obesity markers were associated with increased Qalb 2 decades later [9]. These findings are in line with the expected endothelial damage induced by inflammatory and oxidative effects of chronic DM and support the physiopathological link between vascular risk factors and dementia [9-11].

Adhesion Molecules (ICAM-1, VCAM-1, and sICAM-1)

Endothelial cells express intracellular adhesion molecule 1 (ICAM-1) and vascular cell adhesion molecule 1 (VCAM-
1), which participate in transendothelial migration and endothelial cell activation. ICAM-1 and VCAM-1 were upregulated in peripheral vascular dysfunction related to DM [9]. In a small subgroup of patients with DM, there is a higher CSF level of ICAM-1 and VCAM-1, which correlated positively with Qalb levels, suggesting that endothelial damage related to DM may affect cerebral vessels [9].

The measure of the plasmatic soluble portion of ICAM-1 (sICAM-1) is an independent factor for the onset and severity of white matter disease in patients older than 60 years without a history of neurological disease. In a study with $\mathrm{VaD}$ and $\mathrm{AD}$ patients, these vascular adhesion molecules had higher levels in plasma compared with the control group [13]. 
Plasmatic Inflammatory Markers

Markers such as platelet factor IV, CD40 ligand, homocysteine, and interleukin- 6 are associated with radiological progression of cerebral SVD in patients with lacunar stroke, $\mathrm{VaD}$, and vascular parkinsonism [14]. These findings are in line with the increase in interleukin- 6 and tumor necrosis factor-alpha (TNF- $\alpha$ ) in patients with $\mathrm{VaD}$ compared with late-onset $\mathrm{AD}$ [13]. Basolateral interleukin-6 secretion raises in dyslipidemic patients with $\mathrm{AD}$, with or without vascular risk factors [15]. C-reactive protein levels were associated with reduced verbal fluency in nondementia patients with moderate-to-high cardiovascular risk [16].

Endothelial Function Mediators

Endothelial function regulators and vasodilators, such as endothelin-1 (ET-1) and atrial natriuretic peptide, respectively, are associated with an increased risk of $\mathrm{VaD}$ [17]. In a randomized clinical trial investigating the use of medicinal herbs in diabetic patients with $\mathrm{VaD}$, both treatments (the investigational herb and the use of pioglitazone) decreased ET-1 levels after the intervention with a concomitant increase in plasma nitric oxide [18]. The nitric oxide (NO), known as an "endogenous antiatherosclerotic" agent, is a pivotal mediator of endothelial function, and inhibitors of endothelial NO synthase, such as the asymmetric form of dimethylarginine (ADMA), were investigated in 2 registries $[16,19]$. In the first, ADMA levels are independently associated with silent cerebral infarcts [19]. In the second, ADMA levels were associated with low verbal memory performance in asymptomatic patients with moderate-to-high cardiovascular risk [16].

In TREX1 mutation carriers, a genetic cause of SVD triggered primarily by ED, levels of von Willebrand factor (VWF), and angiopoietin-2 were increased. They were also related to disease activity, mainly in individuals older than 40 years $[20,21]$.

On the other hand, antibodies against heparan sulfate (HS Abs), a glycosaminoglycan present in endothelial cells, which plays an important role in angiogenesis, the integrity of vessels' barrier, and processes of cell adhesion were similar in patients with dementia $(\mathrm{VaD}$ and $\mathrm{AD})$ and controls [22].

\section{Neuropathological Findings}

Neuropathological studies show structural changes in the $\mathrm{BBB}$ of patients with $\mathrm{VaD}$ with an increment in collagen types I and IV and fibrohyalinosis in brain vessels of $\mathrm{VaD}$ subjects [23]. In addition, there is a higher expres-

Endothelial Dysfunction and Vascular

Cognitive Impairment sion of kallikrein 6, whose substrates include fibronectin, fibrinogen, collagen types I and VI, and laminin [24, 25].

Tight junctions form an essential structure for the correct functioning of the $\mathrm{BBB}$, and its main components are claudin and occludin proteins. In individuals with $\mathrm{VaD}$, there is a rise in occludin and claudin expression, suggesting a possible compensatory phenomenon $[26,27]$.

\section{Markers Related to Perfusional and Hemodynamic Changes \\ Angiogenic Factors (HIF-1a, VEGF, TNF- $\alpha$, TGF- $\beta$, \\ NGF, and BDNF)}

Multiple cytokines have been recently studied for their interconnected role in neuroprotective pathways associated with ischemia and hypoxia-induced brain injury. Many of these cytokines have in common a regulatory effect on the secretion of vascular endothelial growth factor (VEGF). VEGF is a cytokine secreted by astrocytes with significant neurotrophic and neuroprotective effects that binds to endothelial cells to regulate angiogenesis and vascular permeability [26-28, 31]. Hypoxia-inducible factor (HIF) is a transcriptor factor that mediates neuroprotective effects related to hypoxia conditioning and ischemia and induces upregulation of VEGF [28]. Nerve growth factor (NGF) and brain-derived neurotrophic growth factor (BDNF) have also demonstrated neuroprotective effects under hypoxia/ischemia-induced brain injury by inducing expression of VEGF, through a pathway dependent on HIF-1a [28]. TNF- $\alpha$ is a cytokine with proinflammatory effects that upregulates transforming growth factor $\beta$ (TGF- $\beta$ ) and induces VEGF production [29]. TGF- $\beta$ has anti-inflammatory effects and also upregulates VEGF [29]. Animal models suggest that TGF- $\beta$ is also associated with amyloidogenesis [29].

Janelidze et al. [9] found higher VEGF levels in CSF of patients with different forms of dementia in comparison with controls, with a slightly higher index of bioactive levels of VEGF among patients with $\mathrm{VaD}$, supporting that concomitant vascular factors may participate in the pathogenesis of neurodegenerative diseases. CSF VEGF correlated positively with Qalb, which is in line with the expected increase in BBB permeability induced by VEGF [9]. However, even subgroups without increased Qalb showed higher CSF VEGF levels, which could mean that upregulation of VEGF may precede BBB dysfunction [9]. The GTC haplotype in the VEGF gene is related to $\mathrm{VaD}$, supporting the pathogenic role of this angiogenic factor [33]. There is an increase in CSF VEGF and TGF- $\beta$ levels in patients with $\mathrm{AD}$ and $\mathrm{VaD}$, supporting a potential role in both forms of dementia [29]. Increased levels of intra- 
thecal TNF- $\alpha$ have also been described among patients with stroke, $\mathrm{AD}$, and $\mathrm{VaD}[29]$.

In contrast, Chakraborty et al. [30] found no significant difference in CSF VEGF levels across patients with $\mathrm{VaD}, \mathrm{AD}$, and controls. Ke et al. [28] also did not find significant differences in intrathecal levels of VEGF or BNDF in patients with ischemic cerebrovascular diseases and VCI compared with controls. There were, however, unexpectedly lower levels of HIF-1a and NGF in pathological groups in comparison with controls, which were hypothesized to be secondary to the limited duration of upregulation of those markers in response to ischemia [28].

In another study, patients with $\mathrm{VaD}$ showed decreased secretion of VEGF by peripheral lymphocytes, similar to what was found in the healthy elderly [32]. AD patients showed a more substantial decrease in VEGF secretion, hypothesized to be potentially due to an inhibitory effect of amyloid- $\beta 42$, which could be compromising angiogenesis and the ability to maintain oxygen and nutrient delivery to the brain in those subjects [32].

Overall, these inconsistent results suggest that angiogenic factors may play a yet incompletely understood role in multiple forms of dementia, including vascular and neurodegenerative pathologies. Studies with VEGF CSF levels in $\mathrm{AD}$ populations have also yielded inconsistent results $[29,30,34]$.

\section{Neuropathological Findings}

$\mathrm{VaD}$ patients have perfusional changes and neovascularization [35]. Burke et al. [36] found a higher length density of hippocampal microvessels in those cases. The vessels were narrower than those without dementia, suggesting an ineffective neoangiogenesis [36].

The white matter hypoperfusion reduces the relation of myelin-associated glycoprotein (MAG) to proteolipid protein 1 (PLP1) [37]. In subjects with $\mathrm{VaD}$, this ratio is decreased [38]. A reduction in MAG:PLP1 values correspond to an elevation in VEGF levels, which associates with a raise in white matter vascular density [37]. However, this increment in VEGF has not been found in subsequent studies [38, 39]. Angiopoietin-like 4 (ANGPTL4) is a protein linked to neovascularization, whose production grows in hypoxia situations [40]. A later study on the brains of $\mathrm{VaD}$ patients showed an increase in ANGPTL4 levels in these individuals [41].

Cerebral hypoperfusion triggers compensatory mechanisms to restore blood flow. Endothelin (ET) is a vasoconstrictive peptide produced by endothelial vascular cells and formed by the endothelin-converting enzyme
(ECE) [42]. Therefore, a decrease in MAG:PLP1 values correlates with a decrease in endothelin-1 (ET-1) levels. However, in $\mathrm{VaD}$ patients, this protective mechanism fails as there is an increase in ET-1 levels and ECE- 1 expression remains normal $[37,43]$.

Other evidence of hemodynamic changes in brains of patients with $\mathrm{VaD}$ is a higher expression of the antithrombotic thrombomodulin in subjects with $\mathrm{VaD}$ [44] and raised levels of the vasodilator dihydroxyeicosatrienoic acid (DHET) [55]. The DHET increment is likewise a compensatory mechanism since patients with the R287Q polymorphism in the soluble epoxide hydrolase gene, the enzyme that produces DHET, have a higher volume of white matter lesions [55].

\section{Vasoreactivity}

Vasoreactivity represents the vascular ability to undergo adaptive changes in response to vasodilatory stimuli, such as metabolic changes and increases in neuronal activity $[1,45]$. Cerebral vasoreactivity (CVR) is strongly dependent on the normal function of the neurogliovascular unit and may indirectly reflect the efficacy of collateral circulation [46]. Studies suggest that cerebral vasoreactivity may already be impaired when resting cerebral blood flow is still within normal ranges, making vasoreactivity markers particularly promising in patients with SVD [1]. Assessment of cerebral vasoreactivity is possible through multiple techniques, such as single-photon emission tomography (SPECT), PET, and transcranial Doppler ultrasound (TCD) [45].

\section{Cerebral Vasoreactivity Measured through TCD}

Assessment of cerebral vasoreactivity through TCD involves the calculation of the percent increase in the mean flow velocity (MFV) of the middle cerebral artery (MCA) in response to different stimuli such as acetazolamide, variation in $\mathrm{CO}_{2}$ levels (induced by hypo- or hyperventilation), and administration of $\mathrm{L}$-arginine. L-Arginine is the precursor of NO, representing a useful tool to assess vasodilation mediated by endothelial cells and executed by vascular smooth muscle cells (VSMC) [47]. On the other hand, direct administration of sublingual nitroglycerin can access vasoreactivity independent of endothelial cells, offering a more direct assessment of VSMC function [48].

Staszewski et al. [45] showed diminished CVR in response to breath-holding maneuvers in patients with lacunar stroke, $\mathrm{VaD}$, and vascular parkinsonism compared with matched controls. CVR was reduced among participants with severe brain atrophy, enlarged perivascular 
spaces, and extensive white matter lesions. Both white matter lesions and CSVD burden score on MRI correlated with CVR [45].

In CADASIL patients, there is a higher MCA resting pulsatility index and increased L-arginine-induced vasoreactivity when compared with controls. These findings imply that large cerebral arteries of CADASIL subjects may present impaired endothelial function, but inconsistent results and methodological concerns argue against this interpretation $[45,46,49,50]$. Other findings reinforce that the degeneration of VSMC from small vessels mediates vascular reactivity impairment in CADASIL $[47,48]$.

\section{Peripheral Vasoreactivity}

Extracerebral Vasoreactivity Measured by Doppler

US Flow-Mediated Dilatation

Brachial artery flow-mediated dilatation (FMD) is a useful marker of extracerebral endothelial function in conduit vessels $[45,48]$. In this technique, the diameter of the brachial artery is measured serially before and after hyperemia induced by deflating a sphygmomanometer cuff distal to the US site. In order to evaluate endothelium-independent vasodilation capacity, NTG can be administered, followed by consecutive measurements of the diameter of the brachial artery [48].

In patients with severe sporadic SVD or $\mathrm{VaD}$, there is a lower FMD when compared with control subjects, in agreement with concomitant findings of reduced CVR $[45,51]$. These findings suggest that in the setting of CSVD, the vasoreactivity impairment may also be found in systemic vessels and could be more easily accessible through peripheral vessels than through cerebral vessels [45]. A positive, though weak, correlation between CVR and FMD was found in CSVD patients, suggesting an association between peripheral and cerebral vasoreactivity dysfunction in this population [45]. Moreover, the significant correlation between FMD and Mini-Mental State Examination (MMSE) implies that ED relates to cognitive impairment [51]. Reduced FMD has also been reported among AD subjects, suggesting that ED may also play a role in neurodegenerative pathologies [51].

In the setting of monogenic SVD pathologies, de Boer et al. [48] employed FMD in patients with RVCL-S and with CADASIL and found reduced dilation only in the former group. Even though VSMC degeneration is a feature of CADASIL, de Boer and colleagues did not find a significant reduction in FMD after nitroglycerin administration in this population, which corroborates the belief that small vessels, rather than conduit vessels, are primarily affected in this pathology [48].

Endothelial Dysfunction and Vascular

Cognitive Impairment
Capsaicin-Induced Dermal Blood Flow Variation

Laser Doppler perfusion imaging can access the dermal blood flow (DBF) variation in response to stimuli such as topical administration of capsaicin, with the advantage of providing information about microcirculatory vessels instead of conduit vessels, as is the case with FMD [48]. de Boer et al. [48] found reduced DBF after capsaicin application in patients with CADASIL, which is hypothesized to be associated with impairment of VSMC relaxation in response to endothelial-independent stimuli.

Forearm Resistance Vessel Function Assessment

This technique involves cannulation of the brachial artery and measures variation of forearm blood flow in response to different vasoactive agents, such as acetylcholine (induces vasodilation dependent on endothelial cells), nitroprusside (NO donor that induces vasodilation independent of endothelial function), and verapamil (directly acts on VSMC independently of endothelial cells or NO) [52]. Moser et al. [52] found a significant association between VSMC function, measured with the administration of verapamil, and performance on neuropsychological tests for initiation and processing speed in a population with early-stage atherosclerotic vascular disease. $\mathrm{Cu}$ riously, the same was not found with endothelial function [52].

\section{Other Markers}

In a study of 149 strokepatients with SVD, $\beta$-amyloid-40 levels were correlated with diffuse SVD disease rather than isolated lacunar strokes [53]. Such findings may represent a direct and vasoconstrictive toxic effect of this peptide against the endothelium, leading to BBB dysfunction [53].

Quantitative evaluation of endothelial progenitor cells also appears to be associated with $\mathrm{VaD}$ and $\mathrm{AD}$. Two markers, CD34 and CD133, are particularly useful in this assessment. CD34 identifies endothelial lineage cells, and CD133 allows the quantification of immature lineages, whereas the double staining of these cells allows a measure of reserve capacity and turnover of endothelial cells [54]. Xiao-dong et al. [54] found a decrease in cell levels with these markers compared with the control group, while patients with $\mathrm{AD}$ showed not only a quantitative reduction in progenitor cells but also a correlation with a worse performance in the MMSE.

In addition, SVD burden has been associated with the increment of serum neurofilament light chain (NfL), as demonstrated by Duering et al. [56]. In this study, serum NfL was related to processing speed performance, focal

Dement Geriatr Cogn Disord 2020;49:365-374 371 
neurological symptoms, and disability in patients with CADASIL and sporadic SVD. As a marker of neuroaxonal damage, increased NfL levels in this population suggest that axonal lesion and neuronal loss might be the ultimate consequence of a broader cascade of pathological events involved in SVD [56].

\section{Conclusions}

This review suggests an intrinsic relationship between $\mathrm{ED}$ and $\mathrm{VCI}$, establishing the endothelium as a pivotal target involved in the pathogenesis of the disease. The biomarkers studied indicate a dynamic and whole-brain process. The BBB has its integrity affected, as demonstrated by the higher CSF/plasma albumin ratio and the increment of collagen types I and IV and fibrohyalinosis. Besides, several inflammatory markers (e.g., interleukin-6, TNF- $\alpha$, and C-reactive protein) are upregulated, also impairing $\mathrm{BBB}$ permeability.

Hemodynamic biomarkers' dynamic, for its part, suggests a state of hypoperfusion (e.g., decrease in MAG:PLP1 ratio) and abnormal vasoreactivity (e.g., abnormal $\mathrm{CO}_{2}$ vasodilation). As a consequence, mechanisms of compensation for ineffective neoangiogenesis (increase in ANGPTL4 and ET-1 levels and decrease in CD34+ and CD133+ progenitor endothelial cells) and antithrombogenicity (higher thrombomodulin levels) would eventually culminate in a vicious cycle of lesion progression. Finally, it is essential to note that the significant heterogeneity of subjects investigated and the multitude of different markers, some with inconsistent results, compromise its application in clinical practice and highlight the importance of establishing this theme as a research priority.

\section{Statement of Ethics}

This research did not involve human participants or animals as this was a systematic review of existing publications, and no primary data were collected. Written informed consent was, therefore, not obtained, and ethical approval was not sought.

\section{Conflict of Interest Statement}

Dr. Pontes-Neto received research support from Conselho Nacional de Desenvolvimento Científico e Tecnológico (CNPq: 402388/2013-5), Fundação de Amparo à Pesquisa do Estado de São Paulo (FAPESP: 2016/15236-8), and CAPES (402388/2013-5). The other authors declare no conflicts of interest.

\section{Funding Sources}

This research received no specific grant from any funding agency in the public, commercial, or not-for-profit sectors.

\section{Author Contributions}

R.K.M.F., M.C.Z., and G.R. carried out the literature search and drafted the manuscript. O.P.N. critically revised the manuscript. All authors read and approved the final version of the manuscript.

\section{References}

1 Wardlaw JM, Smith C, Dichgans M. Small vessel disease: mechanisms and clinical implications. Lancet Neurol. 2019;18(7):684-96.

2 Pantoni L. Cerebral small vessel disease: from pathogenesis and clinical characteristics to therapeutic challenges. Lancet Neurol. 2010 Jul;9(7):689-701.

3 Mok V, Wong A, Tang WK, Lam WW, Fan YH, Richards PS, et al. Determinants of prestroke cognitive impairment in stroke associated with small vessel disease. Dement Geriatr Cogn Disord. 2005;20(4):225-30.

4 Huisa BN, Caprihan A, Thompson J, Prestopnik J, Qualls CR, Rosenberg GA. Longterm blood-brain barrier permeability changes in Binswanger disease. Stroke. 2015 Sep; 46(9):2413-8
5 Shibata M, Yamada S, Kumar Ram S, Calero M, Bading J, Frangione B, et al. Clearance of Alzheimer's amyloid-ss(1-40) peptide from brain by LDL receptor-related protein- 1 at the blood-brain barrier. J Clin Invest. 2000 Dec;106(12):1489-99.

6 Zlokovic BV, Yamada S, Holtzman D, Ghiso J, Frangione B. Clearance of amyloid betapeptide from brain: transport or metabolism? Nat Med. 2000 Jul;6(7):718-9.

7 Snyder HM, Corriveau RA, Craft S, Faber JE, Greenberg SM, Knopman D, et al. Vascular contributions to cognitive impairment and dementia including Alzheimer's disease. Alzheimers Dement. 2015 Jun;11(6):710-7.

8 Moher D, Liberati A, Tetzlaff J, Altman DG; PRISMA Group. Preferred reporting items for systematic reviews and meta-analyses: the PRISMA statement. PLoS Med. 2009 Jul;6(7): e1000097.
9 Janelidze S, Hertze J, Nägga K, Nilsson K, Nilsson C, Wennstrom M, et al. Increased blood-brain barrier permeability is associated with dementia and diabetes but not amyloid pathology or APOE genotype. Neurobiol Aging. 2017;51(2017):104-12.

10 Skoog I. Risk factors for vascular dementia: review. Dement Geriatr Cogn Disord. 1994; 5(3-4):137-44.

11 Carantoni M, Zuliani G, Munari MR, D’Elia K, Palmieri E, Fellin R. Alzheimer disease and vascular dementia: relationships with fasting glucose and insulin levels. Dement Geriatr Cogn Disord. 2000;11(3):176-80.

12 Hao J, Sing K, Yan Y, Hui J, Ding D, Hong Z. Plasma level of sICAM-1 is associated with the extent of white matter lesion among asymptomatic elderly subjects. Clin Neurol Neurosurg. 2009;111:847-51. 
13 Zuliani G, Cavalieri M, Galvani M, Passaro A, Munari MR, Bosi C, et al. Markers of endothelial dysfunction in older subjects with late onset Alzheimer's disease or vascular dementia. J Neurol Sci. 2008;272(1-2):164-70.

14 Staszewski J, Piusińska-Macoch R, Brodacki B, Skrobowska E, Stępień A. IL-6, PF-4, sCD40 L, and homocysteine are associated with the radiological progression of cerebral small-vessel disease: a 2-year follow-up study. Clin Interv Aging. 2018;13:1135-41.

15 Dias HKI, Brown CLR, Polidori MC, Lip GYH, Griffiths HR. LDL-lipids from patients with hypercholesterolaemia and Alzheimer's disease are inflammatory to microvascular endothelial cells: mitigation by statin intervention. Clin Sci. 2015;129(12):1195-206.

16 Miralbell J, López-Cancio E, López-Oloriz J, Arenillas JF, Barrios M, Soriano-Raya JJ, et al. Cognitive patterns in relation to biomarkers of cerebrovascular disease and vascular risk factors. Cerebrovasc Dis. 2013;36(2):98.

17 Holm H, Nagga K, Nilsson ED, Ricci F, Melander $\mathrm{O}$, Hansson $\mathrm{O}$, et al. Biomarkers of microvascular endothelial dysfunction predict incident dementia: a population-based prospective study. J Intern Med. 2017;282(1):94101.

18 Qiang G, Wenzhai C, Huan Z, Yuxia Z, Dongdong Y, Sen Z, et al. Effect of Sancaijiangtang on plasma nitric oxide and endothelin-1 levels in patients with type 2 diabetes mellitus and vascular dementia: a single-blind randomized controlled trial. J Tradit Chin Med. 2015 Aug;35(4):375-80.

19 Pikula A, Böger RH, Beiser AS, Maas R, DeCarli C, Schwedhelm E, et al. Association of plasma ADMA Levels with MRI markers of vascular brain injury: Framingham offspring study. Stroke. 2009;40(9):2959-64.

20 Pelzer N, Bijkerk R, Reinders MEJ, van Zonneveld AJ, Ferrari MD, van den Maagdenberg AMJM, et al. Circulating endothelial markers in retinal vasculopathy with cerebral leukoencephalopathy and systemic manifestations. Stroke. 2017;48(12):3301-7.

21 Stott DJ, Spilg E, Campbell AM, Rumley A, Mansoor MA, Lowe GD. Haemostasis in ischaemic stroke and vascular dementia. Blood Coagul Fibrinolysis. 2001;12(8):651-7.

22 Briani C, Cagnin A, Gallo L, Toffanin E, Varagnolo $\mathrm{M}$, Zaninotto $\mathrm{M}$, et al. Anti-heparan sulphate antibodies and homocysteine in dementia: markers of vascular pathology? J Neurol Sci. 2005;229-30:215-8.

23 Lin JX, Tomimoto H, Akiguchi I, Matsuo A, Wakita $\mathrm{H}$, Shibasaki $\mathrm{H}$, et al. Vascular cell components of the medullary arteries in Binswanger's disease brains: a morphometric and immunoelectron microscopic study. Stroke. 2000 Aug;31(8):1838-42.

24 Ashby EL, Kehoe PG, Love S. Kallikrein-related peptidase 6 in Alzheimer's disease and vascular dementia. Brain Res. 2010;1363:1-10.
25 Lemańska-Perek A, Leszek J, KrzyzanowskaGołab D, Radzik J, Katnik-Prastowska I. Molecular status of plasma fibronectin as an additional biomarker for assessment of $\mathrm{Alz}$ heimer's dementia risk. Dement Geriatr Cogn Disord. 2009;28(4):338-42.

26 Romanitan MO, Popescu BO, Spulber S, Băjenaru O, Popescu LM, Winblad B, et al. Altered expression of claudin family proteins in Alzheimer's disease and vascular dementia brains. J Cell Mol Med. 2010;14(5):1088-100.

27 Romanitan MO, Popescu BO, Winblad B, Bajenaru OA, Bogdanovic N. Occludin is overexpressed in Alzheimer's disease and vascular dementia. J Cell Mol Med. 2007;11(3):56979.

28 Ke XJ, Zhang JJ. Changes in HIF-1a, VEGF, NGF and BDNF levels in cerebrospinal fluid and their relationship with cognitive impairment in patients with cerebral infarction. J Huazhong Univ Sci Technol Med Sci. 2013; 33(3):433-7.

29 Tarkowski E, Issa R, Sjögren M, Wallin A, Blennow K, Tarkowski A, et al. Increased intrathecal levels of the angiogenic factors VEGF and TGF-beta in Alzheimer's disease and vascular dementia. Neurobiol Aging. 2002;23(2):237-43.

30 Chakraborty A, Chatterjee M, Twaalfhoven H, Del Campo Milan M, Teunissen CE, Scheltens $\mathrm{P}$, et al. Vascular endothelial growth factor remains unchanged in cerebrospinal fluid of patients with Alzheimer's disease and vascular dementia. Alzheimers Res Ther. 2018; 10(1):58-7.

31 Ahmed-Jushuf F, Jiwa NS, Arwani AS, Foot P, Bridges LR, Kalaria RN, et al. Age-dependent expression of VEGFR2 in deep brain arteries in small vessel disease, CADASIL, and healthy brains. Neurobiol Aging. 2016;42:110-5.

32 Solerte SB, Ferrari E, Cuzzoni G, Locatelli E, Giustina A, Zamboni M, et al. Decreased release of the angiogenic peptide vascular endothelial growth factor in Alzheimer's disease: recovering effect with insulin and DHEA sulfate. Dement Geriatr Cogn Disord. 2005; 19(1):1-10

33 Kim Y, Nam YJ, Lee C. Haplotype analysis of single nucleotide polymorphisms in VEGF gene for vascular dementia. Am J Med Genet B Neuropsychiatr Genet. 2006;141B(4):3325.

34 Hohman TJ, Bell SP, Jefferson AL. The role of vascular endothelial growth factor in neurodegeneration and cognitive decline: exploring interactions with biomarkers of Alzheimer disease. JAMA Neurol. 2015;72(5):520-9.

35 Baloyannis SJ. Pathological alterations of the climbing fibres of the cerebellum in vascular dementia: a Golgi and electron microscope study. J Neurol Sci. 2007;257(1-2):56-61.

36 Burke MJ, Nelson L, Slade JY, Oakley AE, Khundakar AA, Kalaria RN. Morphometry of the hippocampal microvasculature in poststroke and age-related dementias. Neuropathol Appl Neurobiol. 2014;40(3):284-95.
37 Barker R, Ashby EL, Wellington D, Barrow VM, Palmer JC, Kehoe PG, et al. Pathophysiology of white matter perfusion in Alzheimer's disease and vascular dementia. Brain. 2014;137(Pt 5):1524-32.

38 Thomas T, Miners S, Love S. Post-mortem assessment of hypoperfusion of cerebral cortex in Alzheimer's disease and vascular dementia. Brain. 2015;138(Pt 4):1059-69.

39 Sinclair LI, Tayler HM, Love S. Synaptic protein levels altered in vascular dementia. Neuropathol Appl Neurobiol. 2015;41(4):533-43.

40 Babapoor-Farrokhran S, Jee K, Puchner B, Hassan SJ, Xin X, Rodrigues M, et al. Angiopoietin-like 4 is a potent angiogenic factor and a novel therapeutic target for patients with proliferative diabetic retinopathy. Proc Natl Acad Sci U S A. 2015;112(23):E3030-9.

41 Chakraborty A, Kamermans A, van Het Hof B, Castricum K, Aanhane E, van Horssen J, et al. Angiopoietin like- 4 as a novel vascular mediator in capillary cerebral amyloid angiopathy. Brain. 2018;141(12):3377-88.

42 Shimada K, Matsushita Y, Wakabayashi K, Takahashi M, Matsubara A, Iijima Y, et al. Cloning and functional expression of human endothelin-converting enzyme cDNA. Biochem Biophys Res Commun. 1995 Feb; 207(2):807-12.

43 Palmer JC, Baig S, Kehoe PG, Love S. Endothelin-converting enzyme-2 is increased in Alzheimer's disease and up-regulated by Abeta. Am J Pathol. 2009;175(1):262-70.

44 Giwa MO, Williams J, Elderfield K, Jiwa NS, Bridges LR, Kalaria RN, et al. Neuropathologic evidence of endothelial changes in cerebral small vessel disease. Neurology. 2012;78(3): 167-74.

45 Staszewski J, Skrobowska E, PiusińskaMacoch R, Brodacki B, Stępień A. Cerebral and extracerebral vasoreactivity in patients with different clinical manifestations of cerebral small-vessel disease: data from the significance of hemodynamic and hemostatic factors in the course of different manifestations of cerebral small-vessel disease study. J Ultrasound Med. 2019;38(4):975-87.

46 Migrino RQ, Truran S, Karamanova N, Serrano GE, Madrigal C, Davies HA, et al. Human cerebral collateral arteriole function in subjects with normal cognition, mild cognitive impairment, and dementia. Am J Physiol Heart Circ Physiol. 2018;315(2):H284-90.

47 Peters N, Freilinger T, Opherk C, Pfefferkorn T, Dichgans M. Enhanced L-arginine-induced vasoreactivity suggests endothelial dysfunction in CADASIL. J Neurol. 2008;255(8): $1203-8$

48 de Boer I, Stam AH, Buntinx L, Zielman R, van der Steen I, van den Maagdenberg AMJM, et al. RVCL-S and CADASIL display distinct impaired vascular function. Neurology. 2018; 91(10):e956-63.
Endothelial Dysfunction and Vascular Cognitive Impairment
Dement Geriatr Cogn Disord 2020;49:365-374 DOI: $10.1159 / 000510053$ 
49 Brennan-Krohn T, Salloway S, Correia S, Dong M, De La Monte SM. Glial vascular degeneration in CADASIL. J Alzheimers Dis. 2010;21(4):1393-402.

50 Craggs LJL, Fenwick R, Oakley AE, Ihara M, Kalaria RN. Immunolocalization of plateletderived growth factor receptor- $\beta$ (PDGFR- $\beta$ ) and pericytes in cerebral autosomal dominant arteriopathy with subcortical infarcts and leukoencephalopathy (CADASIL). Neuropathol Appl Neurobiol. 2015;41(4):557-70.
51 Tachibana H, Washida K, Kowa H, Kanda F, Toda T. Vascular function in Alzheimer's disease and vascular dementia. Am J Alzheimers Dis Other Demen. 2016;31(5):437-42.

52 Moser DJ, Miller IN, Hoth KF, Correia M, Arndt S, Haynes WG. Vascular smooth muscle function is associated with initiation and processing speed in patients with atherosclerotic vascular disease. J Int Neuropsychol Soc. 2008;14(4):535-41.

53 Gomis M, Sobrino T, Ois A, Millán M, Rodríguez-Campello A, Pérez de la Ossa N, et al. Plasma beta-amyloid 1-40 is associated with the diffuse small vessel disease subtype. Stroke. 2009;40(10):3197-201.
54 Kong XD, Zhang Y, Liu L, Sun N, Zhang MY, Zhang JN. Endothelial progenitor cells with Alzheimer's disease. Chin Med J. 2011;124(6): 901-6.

55 Nelson JW, Young JM, Borkar RN, Woltjer RL, Quinn JF, Silbert LC, et al. Role of soluble epoxide hydrolase in age-related vascular cognitive decline. Prostaglandins Other Lipid Mediat. 2014;113-5:30-7.

56 Duering M, Konieczny MJ, Tiedt S, Baykara E, Tuladhar AM, Leijsen EV, et al. Serum neurofilament light chain levels are related to small vessel disease burden. J Stroke. 2018 20(2):228-38. 